
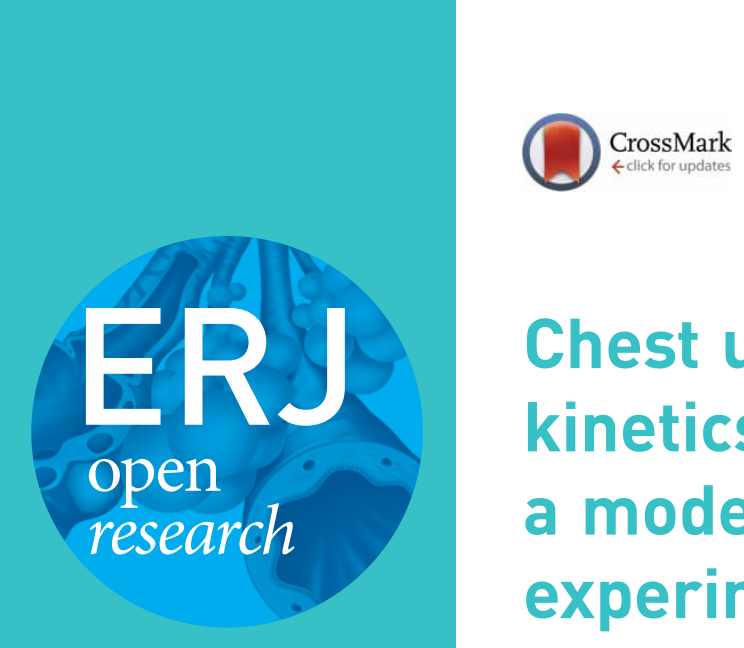

\title{
Chest ultrasonography to assess the kinetics and efficacy of talc pleurodesis in a model of pneumothorax: an experimental animal study
}

\author{
Rachid Tazi-Mezalek (1) ${ }^{1,2}$, Diane Frankel ${ }^{3,4}$, Marc Fortin $^{5}$, Elise Kaspi ${ }^{3,4}$, \\ Julien Guinde ${ }^{1,4}$, Alexandra Assolen ${ }^{4}$, Sophie Laroumagne ${ }^{1,2}$, \\ Andree Robaglia' ${ }^{3,4}$, Herve Dutau', Patrice Roll ${ }^{3,4}$ and Philippe Astoul ${ }^{1,2,6}$
}

Affiliations: ${ }^{1}$ AP-HM, Hôpital Nord, Dept of Thoracic Oncology, Pleural Diseases, and Interventional Pulmonology, Marseille, France. ${ }^{2} \mathrm{G}-$ Echo, French National Group for Chest Ultrasonography, Paris, France. ${ }^{3}$ Aix-Marseille University, INSERM, GMGF, Marseille, France. ${ }^{4}$ AP-HM, Hôpital de la Timone, Service de Biologie Cellulaire, Marseille, France. ${ }^{5}$ Institut Universitaire de Cardiologie et de Pneumologie de Québec, Ville de Québec, Canada. ${ }^{6}$ Aix-Marseille University, Marseille, France.

Correspondence: Philippe Astoul, Hôpital Nord, Dept of Thoracic Oncology, Pleural Diseases and Interventional Pulmonology, Chemin des Bourrely, 13015 Marseille, France. E-mail: pastouldap-hm.fr

ABSTRACT Talc pleurodesis is used to avoid recurrences in malignant pleural effusions or pneumothorax. The lack of lung sliding detected by chest ultrasonography (CUS) after talc application is indicative of the effectiveness of pleurodesis. The objective of our study was to explore, in an animal model, the capacity of CUS to predict the quality of a symphysis induced by talc poudrage (TP) and talc slurry (TS).

We induced an artificial pneumothorax in six healthy pigs prior to talc application. TP was performed on one hemithorax, followed by TS on the other side 1 week later. 108 points on the chest were marked and evaluated by ultrasonography during the study.

TP showed higher sonographic scores compared to TS starting from $72 \mathrm{~h}$ after talc administration. At autopsy, a higher grade of symphysis was observed for TP, and a high correlation rate was registered between CUS and macroscopic findings. Histological analysis also showed a higher grade of pleural symphysis for TP.

CUS is a reliable tool to assess talc pleurodesis. The quality and kinetics of the pleural symphysis are also evaluable by ultrasonography. Pleurodesis by TP is more effective than TS in this experimental model of pneumothorax.

@ERSpublications

Quality of talc pleurodesis and kinetics of pleural symphysis can be evaluated by chest ultrasonography http://ow.ly/8Ejt30jVTjn

Cite this article as: Tazi-Mezalek R, Frankel D, Fortin M, et al. Chest ultrasonography to assess the kinetics and efficacy of talc pleurodesis in a model of pneumothorax: an experimental animal study. ERJ Open Res 2018; 4: 00158-2017 [https://doi.org/10.1183/23120541.00158-2017].

Received: Dec 102017 | Accepted after revision: April 272018

Copyright $\odot E R S$ 2018. This article is open access and distributed under the terms of the Creative Commons Attribution Non-Commercial Licence 4.0. 


\section{Introduction}

The treatment of recurrent malignant pleural effusion or pneumothorax frequently aims to obliterate the pleural cavity to prevent recurrences. Pleurodesis is the symphysis of the pleural space by a fusion of the visceral and parietal pleura. It can be achieved by mechanical abrasion or injection of a sclerosing agent into the pleural space during a thoracoscopy (talc poudrage (TP)) or through a chest tube (talc slurry (TS)) $[1,2]$. Sterile, medical-grade talc has been used as an effective and safe sclerosing agent for a long time $[3,4]$. It induces parietal and visceral inflammation leading to granuloma formation, pleural adhesions and fibrosis [5].

It is difficult to predict the efficacy of pleurodesis early after talc instillation. In malignant pleural effusion, pleural liquid reconstitution after talc application is a manifest sign of recurrence and failure of the pleurodesis. Chest radiography (CXR) demonstrates the recurrence of the effusion but cannot indicate if pleurodesis has occurred or not. Computed tomography (CT) also cannot predict the effectiveness of pleurodesis early after the procedure. However, talc is detected on CT as high-attenuation clusters along the pleura or into the fissure, characteristically described as split pleura sign [6]. Nevertheless, these features do not predict the success of pleurodesis. Conversely, the persistence of an air leak through the chest drain after the procedure is associated with a pneumothorax on imaging (CXR and/or CT) can predict procedure failure. Residual pneumothorax indicates an absence of the lung re-expansion. Finally, CT and CXR indirectly confirm the failure of pleurodesis by the presence of air or liquid in the pleural space, but cannot predict if the symphysis has occurred within the days following the procedure. Finally, pleural thickening and granulomas induced by talc pleurodesis are avid on fluorine-18 fluorodeoxyglucose positron emission tomography (PET), and can mimic neoplastic diseases even 20 years after the procedure [7]. Thus, PET is also not a reliable method for the assessment of talc pleurodesis.

Chest ultrasonography (CUS) has gained popularity in the last decade for the investigation of pleural diseases and the assessment of pleural procedures $[8,9]$. Small amounts of pleural fluid or air can easily be detected by sonography with more accuracy than by CXR [10-12]. In fact, CUS has been proven to be a reliable imaging modality for the evaluation of a wide variety of chest diseases [13]. Normal movement of the parietal pleura over the visceral pleura during the respiration cycle is recognised as the gliding sign [14] or lung sliding sign [11]. The absence of the lung sliding has been used to diagnose pneumothorax, in combination with the repetition of reverberation A lines, the lack of lung pulse and the presence of a lung point [15]. Moreover, identification of pleural thickening as a hypoechoic structure is possible by CUS in the presence or lack of pleural effusion. Pleural thickening and pleural plaques appear as hypoechoic structures typically found along the posterolateral part of the chest. Finally, calcification can occur and appears with posterior acoustic shadows $[16,17]$.

Talc application aims to create a symphysis of the pleural space. Consequently, the sliding of both pleura will be reduced or vanished on CUS examination if the procedure has been successful. CUS has already been demonstrated as an efficient imaging modality for the evaluation of pleurodesis in rabbits [18].

No device and no method are currently available at the bedside to assess directly and to monitor the effects of pleurodesis. The objective of our study was to evaluate, in pigs, the capacity of CUS to predict the quality of a pleurodesis induced by TP and TS, and to compare the effectiveness of both techniques.

\section{Materials and methods}

\section{Study design}

The present study was conducted in compliance with the recommendations of the Federation of European Laboratory Animal Science Associations and was approved by the animal care committee of Aix-Marseille University (Marseille, France). All the animals (pigs) were submitted to a strict veterinary protocol of anaesthesia: use of intramuscular premedication with ketamine and azaperone, intravenous prophylactic cefazolin before the procedure, intravenous propofol $2 \%$ during the procedure and intravenous tramadol before the talc application. During the procedure (thoracoscopy) animals were connected to mechanical ventilation through a veterinary oxygen mask. Systematic monitoring of blood pressure, cardiac pulse and saturation of oxygen was performed. Once the procedure ended, animals were left to recover in an individual enclosure with appropriate temperature, food, toys and veterinary surveillance.

The animals were randomised into two groups. In the first group, TP was performed first on the right side followed by TS on the left side 1 week later. In the second group, the sides were reversed.

Artificial pneumothorax was first induced on the operating table with a 2-mm Boutin's trocar (Novatech SA, La Ciotat, France) under direct CUS guidance and using local anaesthetic (lidocaine 2\%). Successful introduction of air into the pleural space was confirmed audibly and on real-time ultrasonography. Blunt dissection of the chest wall, 7-mm trocar placement and direct-view rigid thoracoscope (Richard Wolf, Reims, France) insertion were performed safely, allowing examination of the pleural cavity. 
For the TP technique, $2 \mathrm{~g}$ sterile French calibrated talc (Stéritalc; Novatech SA) were applied into pleural cavity with a double balloon insufflator (Richard Wolf) as previously described [19, 20]. A 24 Fr chest tube (Vygon, Ecouen, France) was placed apically and connected to progressive wall suction (maximum of $-50 \mathrm{cmH}_{2} \mathrm{O}$ ) for lung expansion. The chest tube was then removed.

For the TS technique, a dilution of $2 \mathrm{~g}$ sterile graded talc was mixed with $50 \mathrm{~mL}$ saline and $5 \mathrm{~mL}$ lidocaine $2 \%$ according to the literature $[1,2]$, and injected through the chest tube which was placed apically and clamped for $1 \mathrm{~h}$. The chest tube was connected to progressive wall suction until the air leak stopped and was then removed.

\section{Thoracic ultrasonographic evaluation}

Nine points on each hemithorax were pre-defined for CUS examination: three points (superior, middle and inferior) along the anterior, mid- and posterior axillary lines (figure 1a). They were tattooed using subcutaneous permanent ink (figure 1b) to allow repetitive ultrasonographic examination during the study. Sonography (EnVisor C HD; Philips, Suresnes, France) was performed by experienced interventional pulmonologists blind to the talc technique used. All nine points were analysed by ultrasonography and classified as pneumothorax, pleural effusion, lung sliding or pleural symphysis (table 1). A total of 108 points of the chest were evaluated by ultrasonography during the study. Pleural thickening was measured in millimetres at the nine pre-defined points for each animal with high-frequency probe in B Mode. CUS was performed before, immediately after (day 0), and at days 1, 3, 14 and 21, on each side. Finally, autopsy was performed after sacrifice with a bolus of propofol and intravenous potassium chloride injection at day 21 according to the recommendations.

\section{Pleurodesis evaluation}

At the autopsy, a macroscopic pleurodesis score, initially proposed by Light et al. [21] and Colt et al. [22], was used to grade the symphysis semiquantitatively (table 2). Investigators who performed the dissection of the pleura were blinded to the procedure previously made. The nine marked points were removed as a block (muscles, connective tissue, parietal pleura, adhesion and visceral pleura with underlying lung parenchyma) after a meticulous dissection in order not to rupture the symphyses that may have occurred.

A microscopic evaluation (histological examination) was performed for three points (superior, middle and inferior) of the midaxillary line as a predefined consensus. A total of 36 points (18 points for each procedure, TP and TS) among the 108 evaluated points of the study were analysed histologically. Tissue specimens were fixed in formalin $4 \%$ and embedded into paraffin. Finely cut sections of $7-\mu \mathrm{m}$ thickness were placed on slides and stained with haematoxylin-eosin and Masson trichrome. The following pathological items were analysed: pleural symphysis, foreign body granuloma, inflammation, presence of talc particles and integrity of mesothelial cell sheet. The pathologists were blind to the procedure performed before and to the ultrasonographic findings.

\section{Statistical analysis}

Statistical analysis was performed with the Prism software (GraphPad Software Inc., San Diego, CA, USA). Significant differences were determined using nonparametric or parametric paired tests (Wilcoxon test or paired t-test, respectively) and Fisher's exact test. A p-value $<0.05$ was considered as significant.

a)

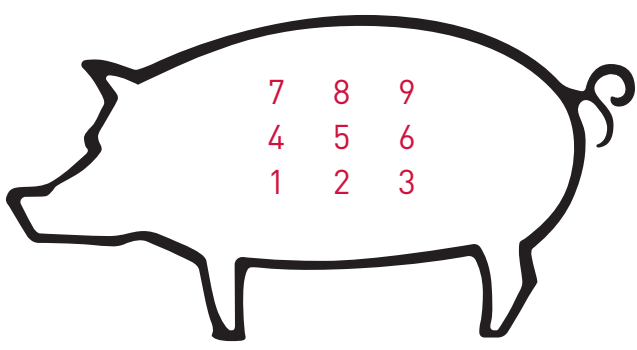

b)

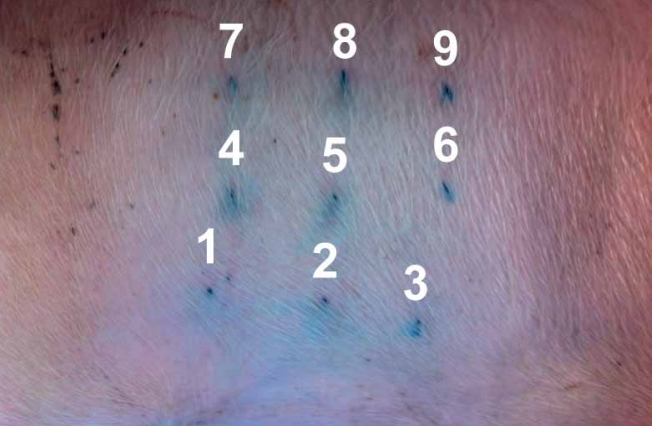

FIGURE 1 Chest ultrasonography design. a) Schematic representation of the nine points on a hemithorax. b) Points tattooed with subcutaneous permanent ink for chest ultrasound follow-up. 


\section{TABLE 1 Grading of sonographic features}

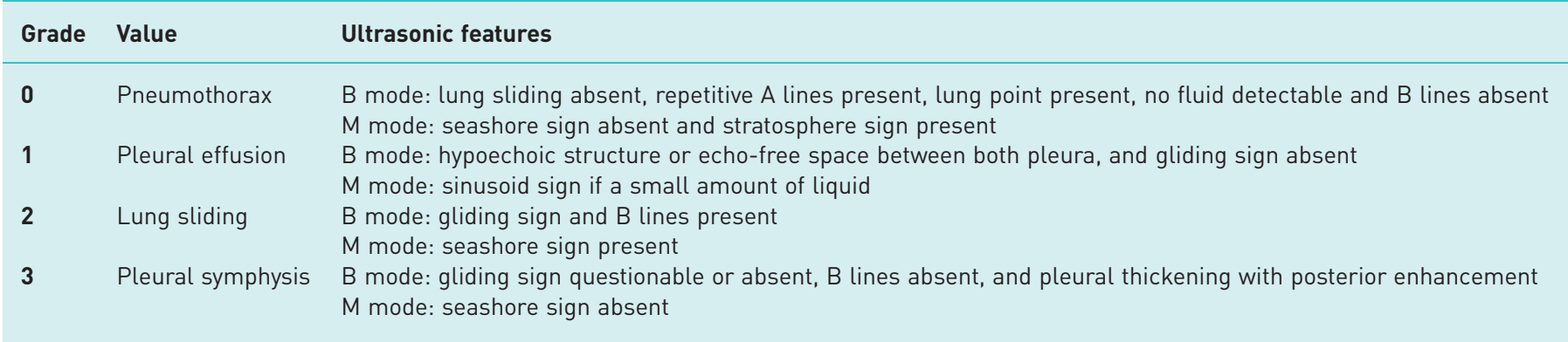

\section{Results}

Six white Landrace pigs of $30 \pm 5 \mathrm{~kg}$ were submitted to both techniques. At baseline, all the pigs had a normal CUS exploration showing evident lung sliding and no area of pleural thickening.

A statistically significant difference in lung sliding between both techniques was observed starting from $72 \mathrm{~h}$ (day 3) after the procedure $(\mathrm{p}=0.005)$ (figure $2 \mathrm{a}$ ). TP achieved a higher sonographic score corresponding to reduced lung sliding (table 1). Symphysis was obtained at most of the points evaluated.

No statistically significant difference was observed between both techniques for pleural thickness except immediately after the procedure. Pleural thickness was higher after TP compared to TS at day $0(p=0.04)$ (figure 2b).

At autopsy (day 21), macroscopic symphysis at the defined points was evaluated during the dissection. Pleural adhesion was rarely observed after TS (figure 3a), whereas pleural adhesions were clearly visible after the TP technique (figure $3 \mathrm{~b}$ ). The macroscopic pleural score was higher for TP than TS ( $\mathrm{p}=0.0011$ ) (figure 3c) and the representation of the pleural adhesions after the dissection showed that macroscopically, TP led to a higher number of pleurodesis areas than TS (figure 3d). Furthermore, we found a positive correlation between the 108 points evaluated by CUS prior to the sacrifice and macroscopic findings $(\mathrm{p}<0.0001, \mathrm{r}=0.55$; Spearman test).

36 points were analysed at the histological level (figure 4). Granulation tissue formations were present in specimens from both techniques. Pleural symphysis was observed in 12 (66.7\%) out of 18 points for TP and in five (27.8\%) out of 18 points for TS with a significant difference ( $\mathrm{p}=0.044$, Fisher test). The fusion between visceral and parietal pleura with a disruption or lack of the mesothelial cells sheet was statistically significant between both techniques ( $\mathrm{p}=0.018$, Fisher test). We found the presence of a mesothelial cell sheet in five (27.8\%) of 18 points for TP and in 13 (72.2\%) of 18 points for TS. No significant differences were observed between the two techniques related to the presence of talc, inflammatory reaction and presence of granulomas (table 3).

For all these results, no difference was shown between the two groups of animals depending on the side of the TP and TS procedures.

\section{Discussion}

The management of malignant pleural effusion and recurrent pneumothorax are common clinical problems. These situations are frequently treated by an attempt to create a pleurodesis with TS or TP. After talc administration, the fusion of the visceral and parietal pleura should prevent the pleural surfaces from gliding over. CUS has recently emerged as an efficient technique to analyse pulmonary and pleural structures. The evaluation of lung sliding after talc application could be indicative of the effectiveness of a pleurodesis. In our study, we used an animal model (pig) to evaluate the capacity of CUS to predict the

\section{TABLE 2 Macroscopic grading score of the symphysis}

\begin{tabular}{ll} 
Grade & Pleurodesis \\
\hline $\mathbf{0}$ & Normal pleural space and lung \\
$\mathbf{1}$ & No adhesion but pleural space inflamed as evidenced by redness and fibrin deposition \\
$\mathbf{2}$ & Few scattered adhesions \\
$\mathbf{3}$ & Generalised scattered adhesions \\
$\mathbf{4}$ & Complete obliteration of the pleural space by adhesions
\end{tabular}



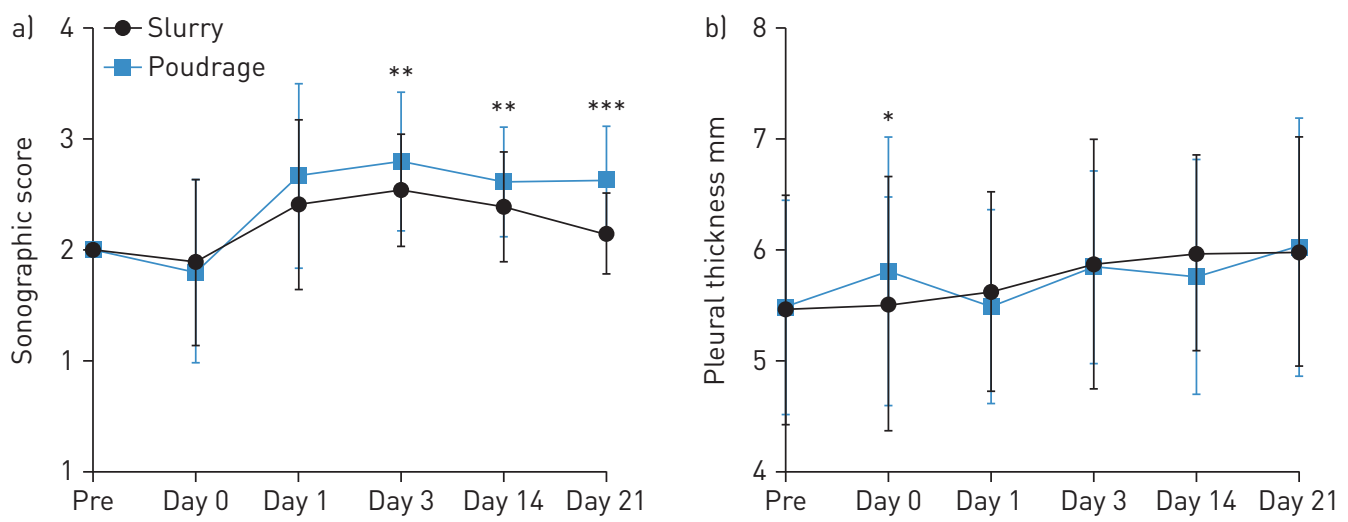

FIGURE 2 Induced pleurodesis depending on the two techniques. a) Comparison of the sonographic score over the study. A statistically significant difference for lung sliding between both techniques is observed starting from $72 \mathrm{~h}$ (day 3) after the procedure. Talc poudrage achieved a higher sonographic score than talc slurry. Day 3: $p=0.005$; day 14: $p=0.0075$; day 21: $p<0.0001$ (Wilcoxon test). b) Comparison of the pleural thickness evaluated by chest ultrasonography over the study. No statistically significant difference in the pleural thickness was observed between the techniques except immediately after the procedure: at day 0 , pleural thickness was higher after poudrage than slurry ( $p=0.04$, paired t-test). ${ }^{*}: p<0.05{ }^{* *}: p<0.01 ;{ }^{* * *}: p<0.001$.

quality of pleurodesis induced by talc after the creation of a pneumothorax and we compared the effectiveness of two technics, TP and TS. We confirm the efficacy of CUS to predict and to easily follow pleurodesis constitution after talc administration. We also identified TP as a superior technique to TS for the induction of pleurodesis in the context of pneumothorax.
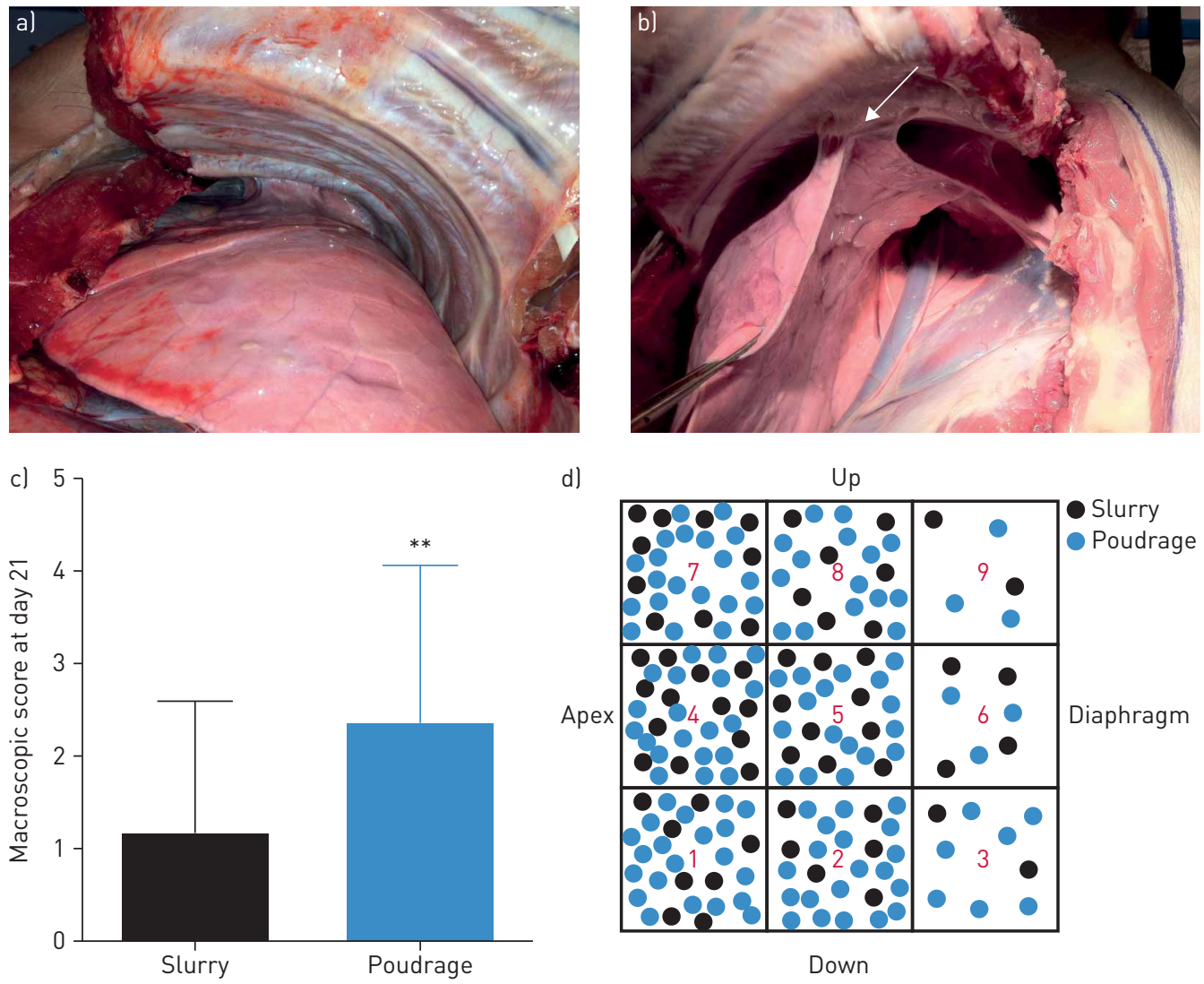

FIGURE 3 Macroscopic view of the pleural space at autopsy. a) After the talc slurry technique, there is no pleural adhesion. b) After the talc poudrage technique, pleural adhesions (arrow) are clearly visible. c) Comparison of the macroscopic score between both techniques. At day 21, the macroscopic pleural score was higher for talc poudrage than talc slurry ( $p=0.0011$. Wilcoxon test). $d)$ Schematic representation of the pleural adhesions after dissection with colour code for the techniques. ${ }^{* *}: p<0.01$. 

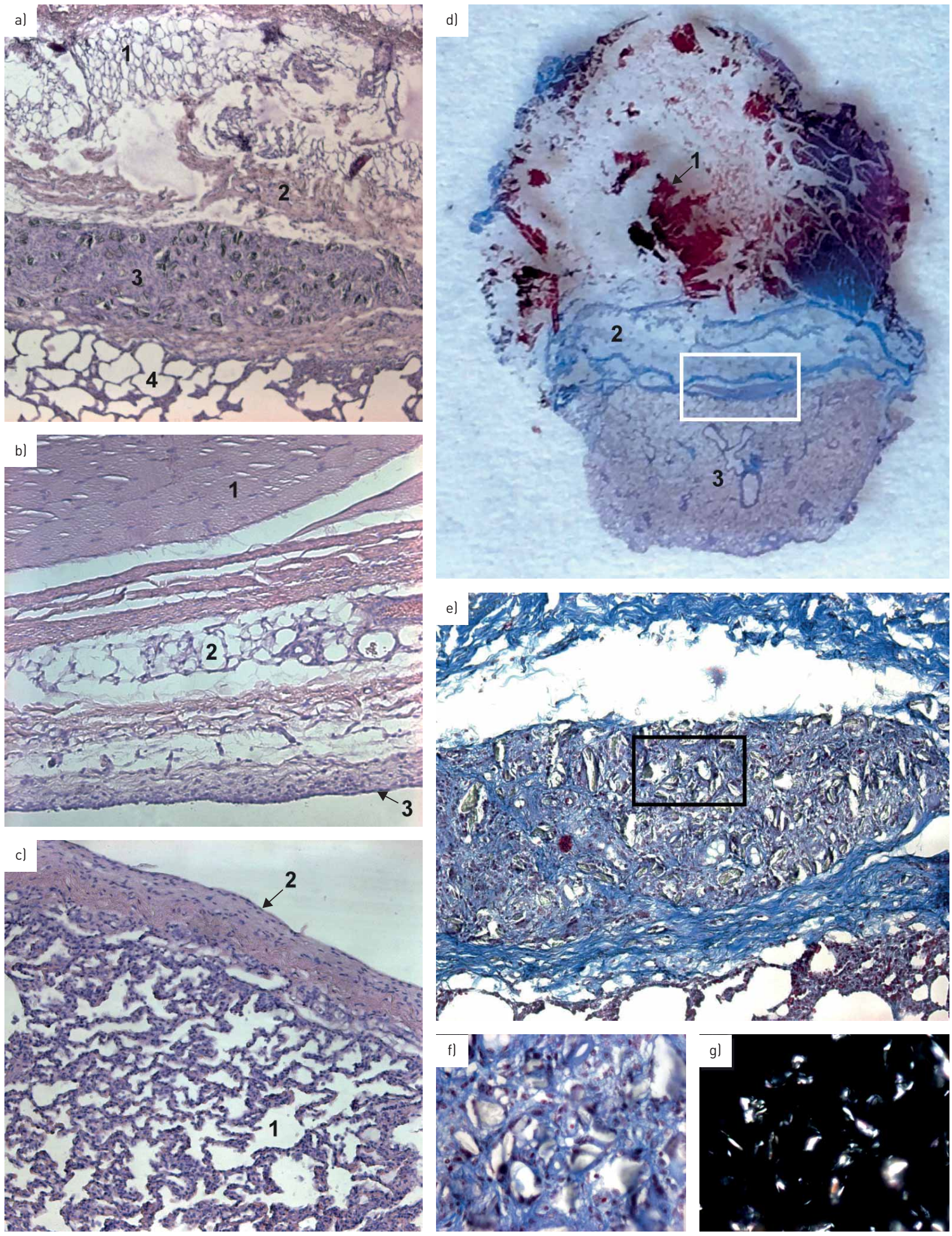

FIGURE 4 Pathological findings. a) Photomicrograph of parietal and visceral symphysis after a talc pleurodesis (haematoxylin-eosin staining, $5 \times$ magnification). 1: chest wall fat tissue; 2: chest wall muscle layer; 3: foreign body granuloma with particle of talc inside; 4: lung parenchyma. Note the absence of differentiation between both pleura and the presence of talc particle with a foreign body reaction. b) Photomicrograph of normal parietal pleura without a sign of adhesion (haematoxylin-eosin staining, 10x magnification). 1: muscle; 2: connective tissue; 3: mesothelial cell sheet of the parietal pleura. Note the integrity of the layer. c) Photomicrograph of normal visceral pleura without signs of adhesion (haematoxylin-eosin staining, 10x magnification). 1: lung parenchyma; 2: mesothelial cell sheet of the visceral pleura. d) Finely cut section of $7 \mu \mathrm{m}$ thickness stained with haematoxylin-eosin representing a pleural symphysis after talc application. 1: muscle; 2: connective tissue; 3 : lung parenchyma. e) Photomicrograph of a foreign body granuloma with particle of talc inside (trichrome staining, 10x magnification), corresponding to the region within the white rectangle in d). f) Photomicrograph of sterile and calibrated talc particle, corresponding to the region within the black rectangle in e). Note the presence of reactive cells all around the particles (trichrome staining, 40x magnification). g) Photomicrograph with polarised filter highlighting the particles of talc. 


\begin{tabular}{lrcc}
\hline \multicolumn{2}{l}{ TABLE 3 Results of histological examination } & & \\
& Talc poudrage & Talc slurry & p-value \\
\hline Pleural symphysis & $12 / 18(66.7 \%)$ & $5 / 18(27.8 \%)$ & $0.044^{*}$ \\
Foreign body granuloma & $12 / 18(66.7 \%)$ & $6 / 18(33.3 \%)$ & 0.094 \\
Inflammation & $16 / 18(88.9 \%)$ & $15 / 18(83.3 \%)$ & 0.99 \\
Presence of talc particles & $14 / 18(77.8 \%)$ & $11 / 18(61.1 \%)$ & 0.47 \\
Presence of mesothelial cell sheet & $5 / 18(27.8 \%)$ & $13 / 18(72.2 \%)$ & $0.018^{*}$ \\
\hline${ }^{*}:$ p<0.05 for talc poudrage versus slurry, Fisher test. & & \\
\hline
\end{tabular}

CXR and CT are usually used as standard techniques to identify pleural diseases. Typically, pleural and extrapleural lesions have obtuse angles with the chest wall, contrary to subpleural processes in the pulmonary parenchyma, in which the angle is more likely to be acute. Pleural plaque and thickening are easily identified as symmetric, smooth and soft-tissue density on CT scans. In recent years, ultrasonography has emerged as a new approach to evaluate chest diseases and it is now the method of choice in the guidance of pleural procedures [13]. It has the advantage of providing real-time imaging of the parietal and visceral pleura, and demonstrating the presence or absence of lung sliding. Sonography is accurate in detecting pleural adhesions. Moreover, its use prior medical thoracoscopy facilitates trocar insertion and prevents visceral injuries [23-25]. Finally, CUS has been proposed to detect pleurodesis after talc application, as demonstrated in animal models by an absence of the lung sliding sign [18].

In our study, during dissection, we observed more macroscopic symphyses at the pre-defined points after TP than TS. Adhesions following TS were logically present in areas near the distal end of the chest tube and in gravity-dependent areas, while adhesions after talc insufflation were more uniform. We believe that obliteration of the pleural space would be more effective if the application of talc is more diffuse and if a larger pleural surface is treated, as induced by TP.

In accordance with several studies [5, 18, 23-26], the present work demonstrates that ultrasonography is an accurate imaging modality to predict pleural adhesions and to identify zones of ineffective pleurodesis. A significant concordance rate between ultrasonographic evaluation and macroscopic findings was found. Consequently, the quality of the symphysis comparing both techniques seems to be nicely assessed by ultrasonography, although more studies are needed to confirm our results.

There is a general agreement that application of talc into the cavity creates diffuse inflammation, an imbalance of pleural coagulation and fibrinolysis, fibroblast proliferation, and collagen production. Pleural mesothelial cells, targeted by the talc, are the cornerstone of the pleurodesis process, releasing multiple molecules such as interleukin-8, transforming growth factor- $\beta$ and fibroblast growth factor [27]. Following talc administration, there is a rapid neutrophil influx into the pleural space and activation of an inflammatory cascade reaction [5, 27]. In our study, a statistically significant difference in the thickness measured by CUS was observed between both talc techniques in favour of TP, but only immediately after the procedure. This may be explained by the insufflation of the talc and its homogenous deposit over the pleural surface, which could produce an immediate diffuse and transient pleural inflammation, detected by ultrasonography as an increase in the pleural thickness.

Furthermore, a higher sonographic score was observed from the third day after talc instillation, corresponding to a persistent and substantial grade of symphysis. Histopathological changes caused by intrapleural talc administration are well described by others but to our knowledge, no study has assessed the chronology of the direct effect of the talc, in particular by using ultrasonography. In our daily practice, for the management of recurrent spontaneous pneumothorax, we rigorously wait until the third day for chest tube removal after talc poudrage, without any evidence-based data. Sonography represents a potential new tool to assess the kinetics of pleural symphysis and to predict the time of chest tube removal. However, more studies are needed to confirm these data.

The dissection shows that macroscopically, TP leads to a higher number of pleurodesis areas in comparison to TS. It is common sense to consider that the distribution of talc particles after insufflation under visual control during a thoracoscopy (TP) is more homogeneous and diffuse than the injection of a solution through a chest tube (TS), and consequently the distribution of the symphysis remains similar. Based on these results, TP is probably more efficient than TS but this hypothesis should be demonstrated in large, randomised studies. 
Moreover, the fusion between the visceral and parietal pleura with a disruption of the mesothelial cell sheet observed in histological analysis was significantly different between both techniques, in favour of TP. However, no significant differences were observed between the two techniques in the presence of talc, inflammatory reaction or the presence of granulomas. The histological findings suggest that pleurodesis has no effect on the lung parenchyma itself. These data are consistent with previous reports of the safety and efficacy of talc pleurodesis [3, 20, 22, 27, 28].

Despite all these encouraging results, there are several limitations to our study. 1) Pleurodesis could not be achieved within a few centimetres of the diaphragm. This may be due to the choice of the lower point of the chest (points 3, 6 and 9) and to the constant movement of the diaphragm with breathing. Not surprisingly, pleural adhesion distribution was less effective close to the diaphragm for both techniques. 2) We chose to reduce the microscopic analysis for 36 of the 108 defined initial points. We believe that microscopic evaluation will not change from one animal to another or between fibrotic and nonfibrotic areas. 3) This experimental study was limited to six animals (both side of the thorax). However, significant and encouraging results were obtained, which may be proved in a bigger cohort. 4) We used healthy pigs and created an artificial pneumothorax to study the pleurodesis, representing an experimental model of pneumothorax taking into account that in such large animals, the thicknesses of the visceral and parietal pleural are similar to the pleura in human, which is not true for small animals as rodents [29]. The same experimental design might be used using a model of malignant pleural effusions. 5) Pleural thickening is measured using a high-frequency probe in B mode. This measure is an operator-dependent datum and it will certainly difficult to evaluate in the malignant pleural effusion model.

Finally, we believe that the results of this experimental study have clinical implications. Indeed, ultrasonography could have multiple applications in humans, such as to demonstrate the quality of a talc pleurodesis, to compare the effectiveness of the sclerosing technique (TP versus TS), to study the kinetic of pleural symphysis and to assess with certainty the time for chest tube removal.

\section{Conclusions}

Sonography is a reliable tool used by pulmonologists to provide real-time imaging guidance for pleural procedures. Talc pleurodesis by poudrage or slurry can be easily assessed and repeated by ultrasonography days after the procedure. The quality of the pleurodesis and kinetics of the pleural symphysis can be evaluated indirectly by ultrasonography. It helps to detect possible recurrences and plans to remove properly the chest tube. Talc pleurodesis by poudrage seems to be a better technique to induce pleurodesis, as observed in our animal study. To confirm our results, comparison of pleurodesis efficacy and quality between slurry and poudrage need now to be compared using CUS in large, randomised clinical studies.

Acknowledgements: We are indebted to S. Berdah and his team (Marie-Ange and Audrey) from the Surgical Teaching and Research Center (CERC, Faculty of Medicine of the North Hospital, Aix-Marseille University, Marseille, France) for their skilled technical assistance during this study.

Conflict of interest: None declared.

\section{References}

1 Light RW, Vargas FS. Pleural sclerosis for the treatment of pneumothorax and pleural effusion. Lung 1997; 175: 213-223.

2 Light RW. Pleural diseases. 3rd Edn. Philadelphia, Lippincott, Williams and Wilkins, 2000.

3 Bethune N. Pleural poudrage: new technique for the deliberate production of pleural adhesion as preliminary to lobectomy. Thorac Surg 1935; 4: 251.

4 Janssen JP, Collier G, Astoul P, et al. Safety of pleurodesis with talc poudrage in malignant pleural effusion: a prospective cohort study. Lancet 2007; 369: 1535-1539.

5 Rodriguez-Panadero F, Montes-Worboys A. Mechanisms of pleurodesis. Respiration 2012; 83: 91-98.

6 Narayanaswamy S, Kamath S, Williams M. CT appearances of talc pleurodesis. Clin Radiol 2007; 62: 233-237.

7 Vandemoortele T, Laroumagne S, Roca E, et al. Positive FDG-PET/CT of the pleura twenty years after talc pleurodesis: three cases of benign talcoma. Respiration 2014; 87: 243-248.

8 O'Moore PV, Mueller PR, Simeone JF, et al. Sonographic guidance in diagnostic and therapeutic interventions in the pleural space. AJR Am J Roentgenol 1987; 149: 1-5.

9 Jones PW, Moyers JP, Rogers JT, et al. Ultrasound-guided thoracentesis: is it a safer method? Chest 2003; 123: $418-423$.

10 Eibenberger KL, Dock WI, Ammann ME, et al. Quantification of pleural effusions: sonography versus radiography. Radiology 1994; 191: 681-684.

11 Lichenstein DA, Menu Y. A bedside ultrasound sign ruling out pneumothorax in the critically ill: lung sliding. Chest 1995; 108: 1345-1348.

12 Soldati G, Testa A, Sher S, et al. Occult traumatic pneumothorax: diagnostic accuracy of lung ultrasonography in the emergency department. Chest 2008; 133: 204-211.

13 Havelock T, Teoh R, Laws D, et al. Pleural procedures and thoracic ultrasound: British Thoracic Society Pleural Disease Guideline 2010. Thorax 2010; 65: Suppl. 2, ii61-ii76. 
14 Targhetta R, Bourgeois JM, Chavagneux R, et al. Ultrasonic signs of pneumothorax: preliminary work. J Clin Ultrasound 1993; 21: 245-250.

15 Volpicelli G, Elbarbary M, Blaivas M, et al. International evidence-based recommendations for point-of-care lung ultrasound. Intensive Care Med 2012; 38: 577-591.

16 Wernecke K. Sonographic features of pleural disease. AJR Am J Roentgenol 1997; 168: 1061-1066.

17 Hallifax RJ, Talwar A, Wrightson JM, et al. State-of-the-art: Radiological investigation of pleural disease. Respir Med 2017; 124: 88-99.

18 Zhu Z, Donnelly E, Dikensoy O, et al. Efficacy of ultrasound in the diagnosis of pleurodesis in rabbits. Chest 2005; 128: 934-939.

19 Tschopp JM, Bintcliffe O, Astoul P, et al. ERS task force statement: diagnosis and treatment of primary spontaneous pneumothorax. Eur Respir J 2015; 46: 321-335.

20 Bridevaux PO, Tschopp JM, Cardillo G, et al. Short-term safety of thoracoscopic talc pleurodesis for recurrent primary spontaneous pneumothorax: a prospective European multicentre study. Eur Respir J 2011; 38: 770-773.

21 Light RW, Wang NS, Sassoon CS, et al. Talc slurry is an effective pleural sclerosant in rabbits. Chest 1995; 107: $1702-1706$.

22 Colt HG, Russack V, Chiu Y, et al. A comparison of thoracoscopic talc insufflation, slurry, and mechanical abrasion pleurodesis. Chest 1997; 111: 442-448.

23 Sasaki M, Kawabe M, Hirai S, et al. Preoperative detection of pleural adhesions by chest ultrasonography. Ann Thorac Surg 2005; 80: 439-442.

24 Wei B, Wang $\mathrm{T}$, Jiang $\mathrm{F}$, et al. Use of transthoracic ultrasound to predict pleural adhesions: a prospective blinded study. Thorac Cardiovasc Surg 2012; 60: 101-104.

25 Cassanelli N, Caroli G, Dolci G, et al. Accuracy of transthoracic ultrasound for the detection of pleural adhesions. Eur J Cardiothorac Surg 2012; 42: 813-818.

26 Leo F, Dellamonica J, Venissac N, et al. Can chest ultrasonography assess pleurodesis after VATS for spontaneous pneumothorax? Eur J Cardiothorac Surg 2005; 28: 47-49.

27 Hartman DL, Antony VB, Hott JW, et al. Thoracoscopic talc insufflation increases pleural fluid IL-8 levels in patients with malignant pleural effusions. Am J Respir Crit Care Med 1994; 149: Suppl. 2, A974.

28 Fraticelli A, Robaglia-Schlupp A, Riera H, et al. Distribution of calibrated talc after intrapleural administration: an experimental study in rats. Chest 2002; 122: 1737-1741

29 Wang NS. Anatomy of the pleura. Clin Chest Med 1998; 19: 229-240. 